\title{
Development of an Evaluation System for Fusarium Resistance in Wheat Grains and Its Application in Assessment of the Corresponding Effects of Fhb1
}

\author{
Xuan Gong, ${ }^{1}$ Xinyao He, ${ }^{2}$ Yuhui Zhang, ${ }^{1}$ Lei Li, ${ }^{1}$ Zhengxi Sun, ${ }^{1}$ Guihua Bai, ${ }^{3}$ Pawan Kumar Singh, ${ }^{2}$ and $\mathrm{Tao} \mathrm{Li}^{1, \dagger}$ \\ ${ }^{1}$ Jiangsu Key Laboratory of Crop Genetics and Physiology, Jiangsu Co-Innovation Center for Modern Production Technology of \\ Grain Crops, Key Laboratory of Plant Functional Genomics of the Ministry of Education, Jiangsu Key Laboratory of Crop \\ Genomics and Molecular Breeding, Yangzhou University, Yangzhou 225009, China \\ ${ }^{2}$ International Maize and Wheat Improvement Center (CIMMYT), 06600 Mexico DF, Mexico \\ ${ }^{3}$ USDA Hard Winter Wheat Genetics Research Unit, Manhattan, KS 66506, U.S.A.
}

\begin{abstract}
Fusarium head blight (FHB) caused by Fusarium species is a globally important wheat disease. Host resistance to FHB is composed of multiple mechanisms, including resistance to initial infection (type I), disease spread (type II), toxin accumulation (type III), kernel infection (type IV), and yield loss (type V), of which the last three have been less studied. Traditionally, the Fusarium-damaged kernel rate (FDK; percentage of Fusarium-infected grains) from point- or spray-inoculated experiments was used as the parameter for type IV resistance, which may be problematic because of the influence of type II resistance. Here we propose a new definition for type IV resistance: that is, the resistance against Fusarium infection expressed in wheat grains that have the same chance in contact with the pathogen, under favorable temperature and humidity for infection. Fhbl confers strong type II resistance, leading to significantly reduced FHB severity and FDK. To investigate the role of Fhbl in type

grain development stages with Fusarium macrospores both in vivo and in vitro. The in vivo experiments with all florets inoculated demonstrated a significant reduction in thousand kernel weight (TKW) in inoculated grains, regardless of their Fhb1 status and developmental stages. Surprisingly, R22W showed more TKW reduction than S22V, which was supported by the scanning electron microscopy observation that confirmed the more severe degradation of starch granules in R22W grains. The in vitro experiments demonstrated that grains from both $\mathrm{R} 22 \mathrm{~W}$ and $\mathrm{S} 22 \mathrm{~V}$ promoted fungal colonization, but no significant difference was found between the two lines. In summary, our results indicated that the proposed type IV evaluation system is effective in determining different grain resistance levels, providing novel tools for FHB resistance breeding. The finding that Fhb1 is not associated with type IV resistance enriches our understanding of this gene.
\end{abstract} IV resistance, a pair of near-isogenic lines, R22W ( $F h b 1$ carrier, resistant in terms of type II resistance) and S22V (non-Fhb1, susceptible), along with eight wheat genotypes differing at $F h b 1$ were inoculated at different
Keywords: evaluation method, Fhbl, Fusarium head blight, grain resistance, Triticum aestivum
Fusarium head blight (FHB) in wheat, mainly caused by the Fusarium graminearum species complex and $F$. culmorum, is a globally important disease. $F$. avenaceum, $F$. langsethiae, and $F$. sporotrichioides are also causal agents of FHB. The pathogens causing FHB are primarily type A and type B trichothecene producers (Foroud et al. 2019). Based on different host reactions to FHB infection, five resistance mechanisms have been proposed: type I for resistance to initial infection, type II for resistance to disease spread within spikes, type III for resistance to toxin accumulation in grains, type IV for resistance to grain infection, and type $\mathrm{V}$ for tolerance to yield loss (Mesterházy 1995). Of these, type II is the best characterized, followed by type I, whereas types III, IV, and V have not been well studied (Buerstmayr et al. 2009; Liu et al. 2009). The Fusariumdamaged kernel rate (FDK) in spray- or point-inoculated experiments

${ }^{\dagger}$ Corresponding author: T. Li; taoli@yzu.edu.cn

Funding: This work was supported by grants from the State's Key Project of R\&D Plan for Breeding of Top-seven Crops (2017YFD0100801), the National Natural Science Foundation of China (31771772), the National Key R\&D Program: Intergovernmental Key Items for International Scientific and Technological Innovation Cooperation (2018YFE0107700), and the Priority Academic Program Development of Jiangsu Higher Education Institutions (PAPD).

*The $\boldsymbol{e}$-Xtra logo stands for "electronic extra" and indicates that two supplementary figures are published online.

The author(s) declare no conflict of interest.

Accepted for publication 17 February 2020.

(C) 2020 The American Phytopathological Society has been associated with type IV resistance and evaluated in many studies (Ágnes et al. 2014; Goral et al. 2019; Szabó-Hevér et al. 2012; Tamburic-Ilincic 2012), but knowledge on the resistance of wheat grain to FHB, as well as its relationship with other resistance types (especially type II resistance), is insufficient.

The evaluation of type IV resistance is currently based mostly on FDK, which is calculated from grain samples harvested from single spikes, plants, or plots in spray- or point-inoculated experiments as the percentage of Fusarium-infected or affected grains. In most studies, quantitative trait loci (QTLs) for FDK coincided with those for type I and/or II resistance (Buerstmayr et al. 2009; Schweiger et al. 2016; Yang et al. 2005), implying that FDK may not be a good parameter for type IV resistance (equivalent to "grain resistance" in this article), which should be independent from type I and II resistance. Based on its definition, type IV resistance should reflect the resistance of grain per se upon Fusarium infection: that is, the resistance level expressed in wheat grains that have the same chance in contact with the pathogen, under favorable temperature and humidity for infection. Therefore, it is inappropriate to use FDK as the parameter for type IV resistance in spray- or pointinoculated experiments.

Wheat grain is mainly composed of endosperm, which in turn is mainly composed of starch and protein. Two types of starch granules (large and small) were found in wheat endosperm, with diameters of 10 to $30 \mu \mathrm{m}$ for the former and $<10 \mu \mathrm{m}$ for the latter (Parker 1985). Upon Fusarium spp. infection, grains become shriveled and bleached, with poor grain filling and reduced grain weight, owing to the decomposition of grain starch and protein caused by Fusarium spp. The pathogen first infects the exocarp and then the endocarp and finally destroys the amyloplasts and protein in the endosperm (Jansen et al. 2005), leading to a significantly reduced protein content in infected grains. With scanning electron microscopy (SEM), Packa et al. (2008) observed a large amount of hyphae on the surface and in the 
endosperm of infected grain, with small starch granules disappearing and large starch granules being severely damaged.

The Fhbl gene initially identified in Sumai3 is the most stable and effective resistant gene against FHB. It has been reported that wheat genotypes with Fhb1, such as Sumai3, Ning7840, and Wangshuibai (WSB), showed a significantly lower percentage of infected spikelets and FDK than those without Fhb1 (Balut et al. 2013; Clark et al. 2016). However, it is unclear whether the reduced FDK was caused directly by grain resistance or indirectly by low FHB severity or whether Fhbl confers grain resistance. The main objectives of this study were to develop an evaluation system for Fusarium resistance in wheat grain and to apply it in the assessment of the role of Fhbl in grain resistance via both in vivo and in vitro experiments on wheat genotypes differing at $F h b 1$.

\section{Materials and Methods}

Plant materials. Chinese FHB-resistant cultivar Ning7840, U.S. FHB-susceptible cultivar Clark, and two near-isogenic lines (NILs) contrasting in Fhbl alleles (R22W and S22V) were used in this study. Ning7840, an Fhbl carrier, has a pedigree of Anhui11/ Aurora//Sumai3 and consistently shows low FHB severity $(<15 \%)$ under point-inoculated experiments. Clark does not have $F h b 1$ and consistently shows a high level of FHB severity (up to 100\%) under point-inoculated experiments. The two isogenic lines were derived from the cross Ning7840/Clark*7 and generated via markerassisted selection, of which $\mathrm{R} 22 \mathrm{~W}$ has $F h b 1$ and S22V does not. In addition to these four genotypes, several other genotypes were also included in the SEM experiment: (i) Fhbl carriers, including a Japanese variety Tokai66, a Chinese wheat variety Sumai3, and a Chinese wheat landrace WSB; and (ii) non-Fhbl carriers, including a Chinese wheat landrace Haiyanzhong (HYZ), a breeding line S42 that has good type II resistance, and a U.S. wheat cultivar Bobwhite susceptible to FHB.

Fungal isolates and inoculum preparation. A virulent $F$. graminearum isolate of the 15-acetyl-deoxynivalenol (15ADON) chemotype, Fg0865 (kindly provided by Dr. Hongxiang Ma, Jiangsu Academy of Agricultural Sciences, Nanjing, China), was used in both in vivo and in vitro experiments. An additional $F$. graminearum isolate of the 15ADON chemotype, FgW (kindly donated by Dr. Saihua Chen, Yangzhou University, Yangzhou, China), was used only in in vitro experiments.

Pure $F$. graminearum isolates were taken with an inoculation loop from a marginal colony on slant medium and were then transferred onto potato dextrose agar (PDA), which was incubated for 5 to 7 days under $26^{\circ} \mathrm{C}$ and was then kept in a refrigerator under $4^{\circ} \mathrm{C}$ for subsequent use.

Eight to ten fungal plugs were taken from the PDA medium and transferred into a flask with $80 \mathrm{ml}$ of autoclaved liquid mung bean medium, which was then put on a shaker and shaken at $180 \mathrm{rpm}$ un$\operatorname{der} 26^{\circ} \mathrm{C}$ for 72 to $120 \mathrm{~h}$. The spore concentration was measured with a hemacytometer and adjusted to a final concentration of $1 \times 10^{5}$ spores $/ \mathrm{ml}$.

The in vivo experiment. Field experiments and sampling. All wheat materials were sown in the experimental field of Yangzhou University. Each genotype was planted in two replications, each having five rows of 15 plants. The middle florets of a wheat spikelet are usually smaller and less developed than the lateral florets; therefore, at anthesis, all middle florets were manually removed to retain only two lateral florets in each spikelet to ensure that no florets were missed when inoculation was performed. Ten microliters of inoculum was injected into each of the remaining florets of a spike at three developmental stages: 5,10 , and 15 days post-anthesis (dpa), respectively. At 15 days after inoculation, the inoculated spikes were harvested, threshed, and stored at $-20^{\circ} \mathrm{C}$ for subsequent experiments. Uninoculated control spikes had the same treatments except for inoculation, and samplings were carried out at 20, 25, and $30 \mathrm{dpa}$, respectively, corresponding to the sampling timepoints of their inoculated counterparts.

Sample photography. Photographs of the inoculated and check wheat spikes were captured with a Canon G12 camera, and those of the corresponding grains were taken with an MVX10 macrozoom fluorescence microscope. For SEM observation, wheat grain samples were fixed in $2.5 \%$ glutaraldehyde under $4^{\circ} \mathrm{C}$ for $12 \mathrm{~h}$, washed three to four times with $0.1 \mathrm{M}$ of phosphate-buffered saline, and then dehydrated with gradient concentrations of ethanol $(30 \rightarrow$ $50 \rightarrow 70 \rightarrow 80 \rightarrow 90 \rightarrow 95 \rightarrow 100 \%$ ) and a step of anhydrous $\mathrm{Na}_{2} \mathrm{SO}_{4}$, with each step lasting 10 to $15 \mathrm{~min}$. The samples were then dried in a CPD-300 Critical Point Dryer, crosscut, attached to the sample stage with double-sided tape, and finally sprayed with gold nanoparticles in an SCD500 ion sputter coater. Observation of grain starch granules was carried out with SEM (Philip XL-30ESM), and photos were taken at the same magnification.

The in vitro experiment. Mature grain samples were disinfected with $75 \%$ of ethanol for $1 \mathrm{~min}$, washed three times with sterile water, treated with sodium hypochlorite solution for $40 \mathrm{~min}$, and washed three to five times with sterile water for the in vitro assay.

The disinfected grains were arranged on PDA medium in three circles spaced $2 \mathrm{~cm}$ apart, each having six grains. A fungal plug of $F$. graminearum was placed onto the center of the PDA medium, which was then sealed and cultivated under $26^{\circ} \mathrm{C}$. A PDA plate with only a fungal plug in the center was used as the control. Fungal colonies were photographed every $24 \mathrm{~h}$ after $36 \mathrm{~h}$ of initial cultivation in order to record their growth speed. Colony diameter was measured with a vernier, and the time for a colony to cover the entire medium surface was also recorded.

\section{Results}

Changes in spikelets and grains after inoculation. Artificial point-inoculation on all of the remaining lateral florets of each spikelet provided equal chances for every developing grain to contact Fusarium inoculum. Compared with the checks, all of the inoculated florets showed FHB symptoms. Regardless of their allelic status at $F h b 1$, the inoculated spikes were thinner than their respective checks, especially those inoculated at 5 dpa (Supplementary Fig. S1).

Compared with the checks, all grains from the inoculated spikes were markedly shriveled, especially those from spikes inoculated at $5 \mathrm{dpa}$, whereas the shrinkage became less pronounced when inoculated at later stages (Fig. 1). No significant difference was found between Fhbl carriers (Ning7840 and R22W) and non-Fhbl carriers (Clark and S22V) (Fig. 1).

Over the course of grain development, the impact of Fusarium infection kept decreasing in the tested lines, as reflected in the change rate of grain length, width, and thousand kernel weight (TKW). Overall, the most significant change was found for TKW, especially when inoculated at later stages $(15 \mathrm{dpa})$, followed by grain width and grain length (Table 1). No significant difference was observed for grain length among the four tested lines compared at the same developmental stages. Notably, TKW reduction in R22W was significantly greater than that in S22V, demonstrating the negative effect of Fhb1 on grain resistance to Fusarium infection, which is in sharp contrast to its role in type II resistance.

Changes in grain starch granules after Fusarium inoculation. Compared with the uninoculated grains, the inoculated grains showed degradation of starch granules at various degrees (i.e., the more developed the grains, the less degraded the starch granules) (Figs. 2, 3, and 4). At the same developmental stages, however, the tested lines showed different degrees of degradation of starch granules. Clear changes were observed in inoculated grains, including degradation in the kernel protein matrix, reduction in the number of small starch granules, and the pits or cavities on the surface of large starch granules, especially in the equatorial groove region.

When inoculated at $5 \mathrm{dpa}$, significant degradation of starch granules was observed in all tested materials, reflected in sharp shrinkage of and numerous cavities on starch granules compared with the checks (Fig. 2). Among the FHB-resistant cultivars with Fhbl, Ning7840 showed the least degradation in starch granules. Of the FHB-susceptible cultivars, Bobwhite showed the most degradation and almost no starch granules could be observed, whereas Clark exhibited relatively less degradation. Between the two NILs, R22W 
showed markedly more degradation than S22V in which only small pits appeared on the starch granules.

When inoculated at $10 \mathrm{dpa}$, starch granules of the tested genotypes showed various degradation with pits or cavities on the surface, resulting from the invasion of mycelia, whereas those of the checks were intact with a smooth surface (Fig. 3). Of the Fhbl carriers, Ning7840 was the least affected, whereas Sumai3 showed more severe degradation of starch granules. Of the non-Fhbl carriers, HYZ was the least affected and performed better than the Fhbl carriers, followed by S42, which was worse than Ning7840 but better than the other Fhbl carriers. Bobwhite was the worst performer in terms of starch granule degradation, followed by Clark. Of the two NILs, S22V showed similar starch granule degradation as $\mathrm{HYZ}$, whereas $\mathrm{R} 22 \mathrm{~W}$ exhibited much more severe degradation.

When inoculated at $15 \mathrm{dpa}$, starch granules of HYZ and S22V showed a smooth surface without obvious pits or cavities. Obvious degradation of starch granules was still observed in Bobwhite, whereas only small pits were found on starch granules of the other genotypes (Fig. 4).

When the results from different treatments were taken together, Ning7840 always showed the least degradation of starch granules among the Fhb1 carriers, whereas HYZ and S22V were the least affected among the non-Fhbl carriers, in contrast to the severe infection in Bobwhite. Between the two NILs, the Fhb1 carrier R22W always exhibited more severe degradation of starch granules than the non-Fhb1 carrier S22V. These results demonstrated a clear difference in grain resistance to Fusarium infection among the tested genotypes, of which Ning7840, HYZ, and S22V showed good grain resistance, indicating the independence of type IV from type II resistance and a lack of obvious effect of Fhbl on grain Fusarium resistance.

Influence of wheat grains on the growth rate of $F$. graminearum strains. Compared with the check, fungal colonies of all treatments with wheat grains grew much faster; however, no significant difference was found among the three treatments with the same $F$. graminearum strain, implying that all wheat grains, despite their origin from the resistant or the susceptible NIL, can stimulate mycelium growth (Fig. 5; Supplementary Fig. S2). However, under the same treatment, the growth rate of the Fg0865 strain was slower than that of $\mathrm{FgW}$. In terms of time requirements for the fungal colonies to cover the entire Petri dish, $108 \mathrm{~h}$ was needed for Fg0865 compared with only $84 \mathrm{~h}$ for $\mathrm{FgW}$ when wheat grains were added, whereas 132 h was needed for Fg0865 compared with only 108 h for $\mathrm{FgW}$ when grains were not added.

\section{Discussion}

Of the FHB resistance mechanisms, types I and II proposed by Schroeder and Christensen (1963) and type III by Mesterházy (1995) and Mesterházy et al. (1999) had clear definitions and have been widely accepted; however, type IV resistance was not clearly defined by Mesterházy (1995), and no specified evaluation method was proposed either. Thereafter, researchers and breeders usually took FDK as the parameter for type IV resistance, for which grains harvested from point- or spray-inoculated spikes were evaluated to calculate or estimate the percentage of shriveled, bleached, or scabbed grains. In most published studies, the percentage of scabbed spikelets (PSS) as a parameter for type II resistance exhibited a significantly positive correlation with FDK, and most QTLs for PSS coincided with those for FDK (Abate et al. 2008; Bonin and Kolb 2009; Islam et al. 2016; Yang et al. 2005), indicating that FDK is just another parameter for type II resistance instead of type IV resistance, which should be independent from the former. Here we proposed a new definition for type IV resistance: that is, the resistance against Fusarium infection expressed in wheat grains that have the same chance in contact with the pathogen, under favorable temperature and humidity for infection. Based on this definition, we investigated the relationship between $F h b 1$ and grain resistance.

Fhbl is a well-known FHB resistance QTL, consistently showing major effects on type II resistance in different genetic backgrounds.

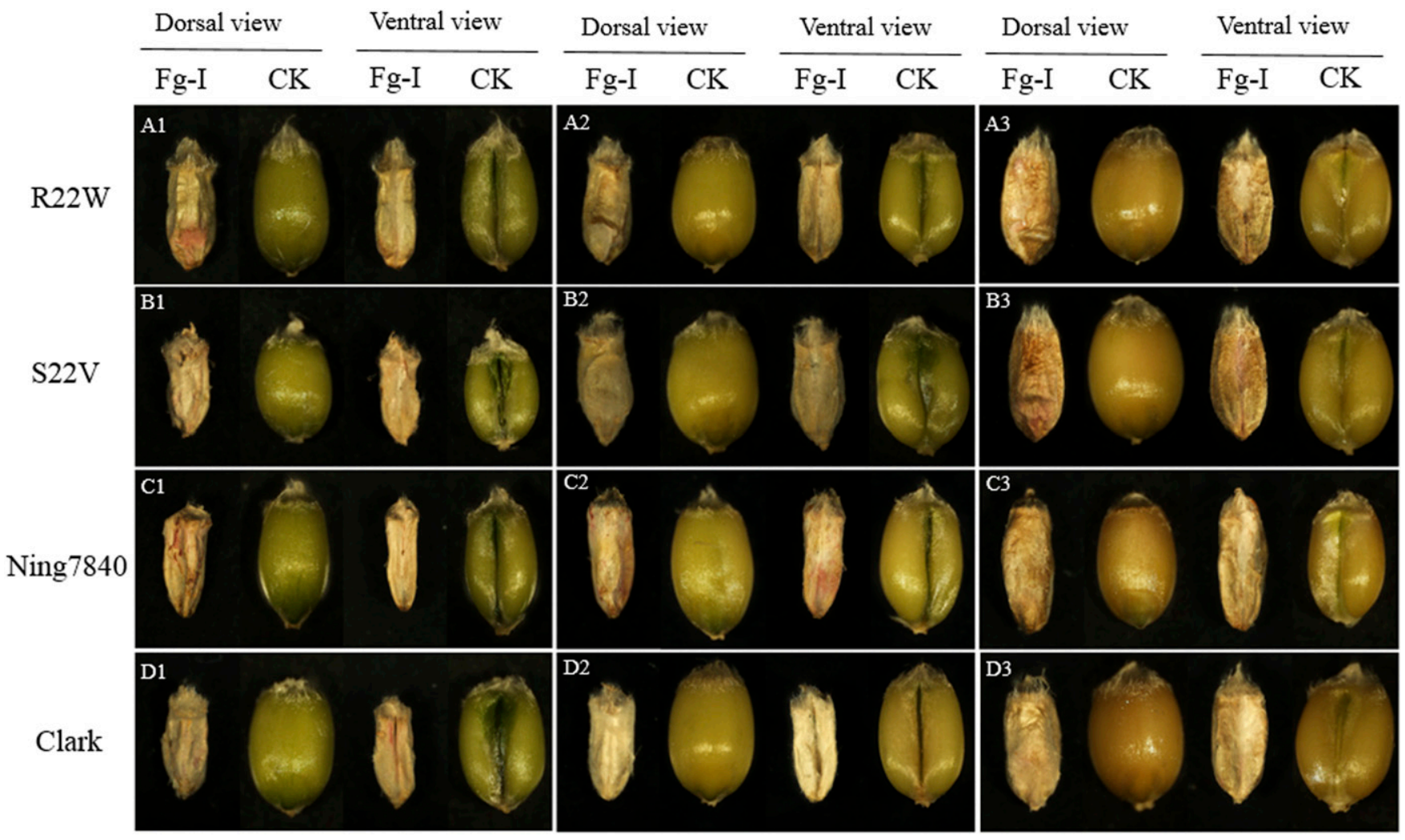

Fig. 1. Phenotypic variation of genotypes inoculated at different developmental stages. All genotypes were inoculated at 5, 10, and 15 days post-anthesis (images 1,2 , and 3 respectively) and grains were all sampled at 15 days postinoculation. A1, A2, and A3, R22W. B1, B2, and B3, S22V. C1, C2, and C3, Ning7840. D1, D2, and D3, Clark. Fg-l, Fusarium graminearum inoculated; CK, uninoculated check. 
Fhbl is located on chromosome arm 3BS and present in Sumai3 and some Chinese and Japanese landraces (Bai et al. 2018; Su et al. 2019). Several studies proposed its role in reducing toxin accumulation, which is still in dispute (Audenaert et al. 2013; Hofstad et al. 2016; Lemmens et al. 2008; Walter et al. 2015). Three articles have been published on the cloning of Fhbl (Li et al. 2019; Rawat et al. 2016; Su et al. 2019); however, none of them revealed its molecular mechanism for the various FHB resistance components, and there have been controversies regarding the candidate genes and their possible roles in FHB resistance. As stated earlier, FDK is a parameter for type II resistance. Thus, it is no wonder that FDK can be significantly reduced by $F h b 1$ as a gene for type II resistance; however, whether this gene provides type IV resistance still remains unclear.

Table 1. Relative change rates of grain length (GL), grain width (GW), and thousand kernel weight (TKW) in wheat lines inoculated at different developmental stages ${ }^{y}$

\begin{tabular}{llrll}
\hline Timing of inoculation $^{\mathbf{z}}$ & Accession & GL & GW & TKW \\
\hline $5 \mathrm{dpa}$ & R22W & $-22.37 \mathrm{a}$ & $-40.00 \mathrm{ab}$ & $-89.09 \mathrm{a}$ \\
& S22V & $-20.27 \mathrm{a}$ & $-47.70 \mathrm{a}$ & $-87.80 \mathrm{~b}$ \\
& Ning7840 & $-18.97 \mathrm{a}$ & $-35.09 \mathrm{~b}$ & $-86.50 \mathrm{c}$ \\
& Clark & $-15.18 \mathrm{a}$ & $-39.22 \mathrm{ab}$ & $-87.11 \mathrm{bc}$ \\
& R22W & $-6.91 \mathrm{a}$ & $-37.78 \mathrm{a}$ & $-77.24 \mathrm{ab}$ \\
& S22V & $-10.05 \mathrm{a}$ & $-36.96 \mathrm{a}$ & $-75.48 \mathrm{~b}$ \\
& Ning7840 & $-5.90 \mathrm{a}$ & $-37.18 \mathrm{a}$ & $-78.50 \mathrm{a}$ \\
$15 \mathrm{dpa}$ & Clark & $-3.44 \mathrm{a}$ & $-27.38 \mathrm{~b}$ & $-76.93 \mathrm{ab}$ \\
& R22W & $0.68 \mathrm{a}$ & $-29.45 \mathrm{a}$ & $-63.62 \mathrm{a}$ \\
& S22V & $2.95 \mathrm{a}$ & $-29.83 \mathrm{a}$ & $-58.49 \mathrm{c}$ \\
& Ning7840 & $4.26 \mathrm{a}$ & $-20.46 \mathrm{a}$ & $-49.73 \mathrm{~d}$ \\
& Clark & $2.90 \mathrm{a}$ & $-23.89 \mathrm{a}$ & $-60.36 \mathrm{~b}$ \\
\hline
\end{tabular}

${ }^{\mathrm{y}}$ Relative change rates are given in percentages and were calculated via $(\mathrm{Vt}-$ $\mathrm{Vck}) / \mathrm{Vck} \times 100$, where $\mathrm{Vt}$ and Vck represent trait values of the treatment and the check, respectively. Different letters following the trait values indicate significance at $P<0.05$.

${ }^{\mathrm{z}}$ dpa, days post-anthesis.
In this study, a pair of NILs for Fhbl and genotypes with a wide range of FHB resistance were used in in vivo and in vitro experiments, in which grain resistance was investigated in different aspects and the results clearly showed that $F h b 1$ does not confer grain resistance.

In the in vivo experiment, we developed a new methodology for evaluating type IV resistance, in which the middle florets as well as the underdeveloped spikelets were eliminated to retain only the well-developed lateral florets. This method ensures the homogeneity in inoculation, providing an equal opportunity for each grain in contact with the inoculum and making the results among grains more comparable. It is well acknowledged that grains become shriveled and of low TKW after Fusarium infection, which was witnessed in our experiments. Based on the changes in grain length, grain width, and TKW after inoculation, we found that grain resistance was influenced by grain developmental stages, with more developed grains having better resistance to Fusarium species, and this was irrelevant to the allelic status of Fhbl. Of the two NILs, R22W with Fhb1 constantly exhibited higher TKW reduction compared with S22V without $F h b 1$, which was unexpected. Mesterházy (1995) also proposed type $\mathrm{V}$ resistance as a measurement for tolerance to yield reduction after Fusarium infection, but few studies to date have targeted this type of resistance. Based on our results, TKW was the only significantly changed trait in relation to grain resistance, which has no relation to the other two key elements of yield (i.e., spikes per unit area and grain number per spike). Therefore, we propose that type $\mathrm{V}$ be merged into type IV resistance because they essentially reflect the same resistance mechanism, and the reduction in TKW under the grain resistance assay proposed in this study could be used as the evaluation parameter for type IV resistance. This simple and feasible methodology can be easily adopted by breeders and researchers.

Parker (1985) demonstrated that large starch granules appear in 4 to $6 \mathrm{dpa}$ in endosperm cells, and the plasma membrane of the starch granules begins to form vesicles or protrusions toward the cell matrix at 12 to $14 \mathrm{dpa}$; the latter were finally isolated from the large starch granules, forming small starch granules, which are able to proliferate
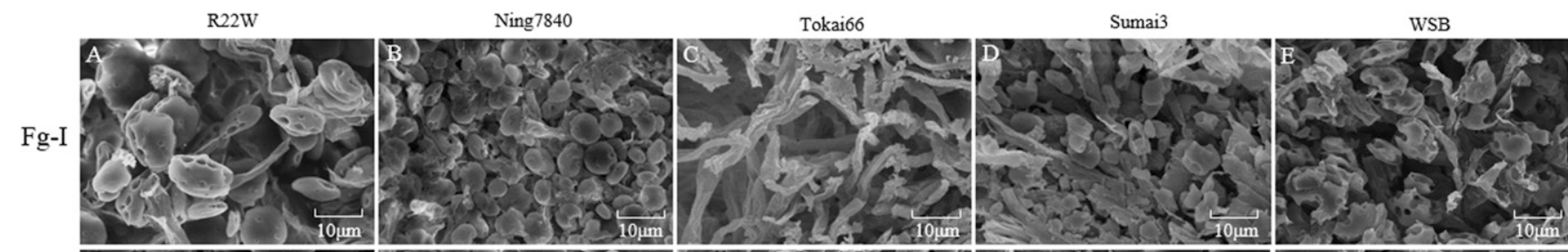

$\mathrm{CK}$
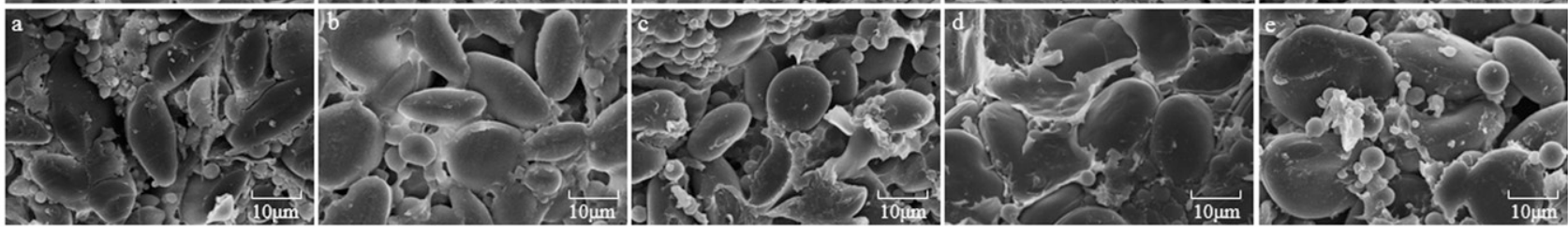

$\mathrm{S} 22 \mathrm{~V}$

Clark
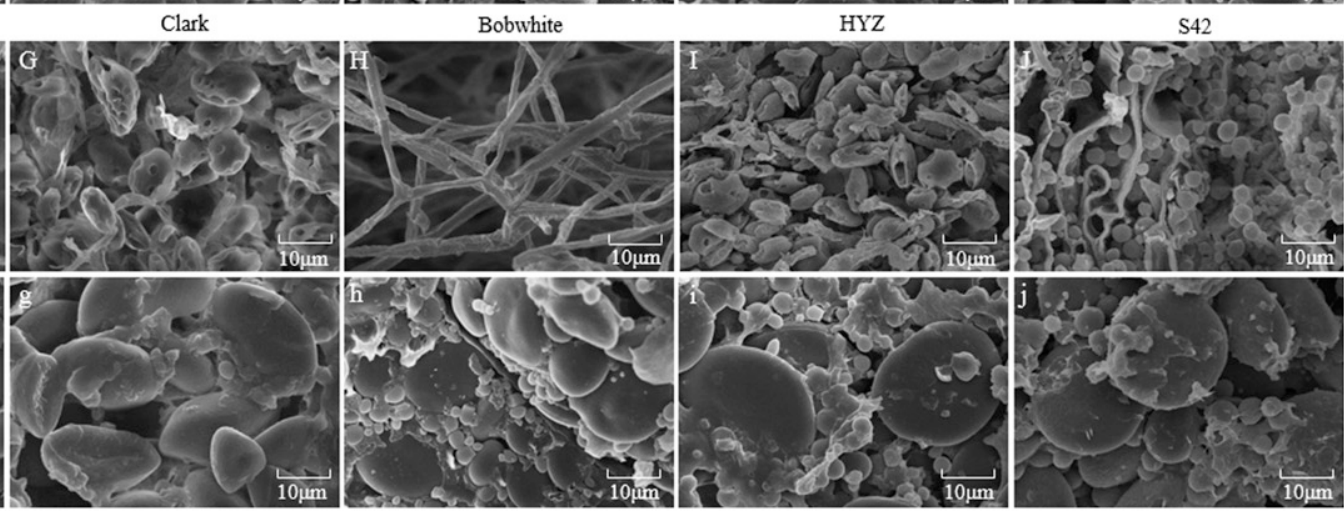

Fig. 2. Scanning electron microscopy of endosperm structures of inoculated genotypes sampled at 20 days post-anthesis. A and a, R22W. B and b, Ning7840. C and c, Tokai66. D and d, Sumai3. E and e, Wangshuibai (WSB). F and f, S22V. G and g, Clark. H and h, Bobwhite. I and i, Haiyanzhong (HYZ). J and j, S42. Fg-l, Fusarium graminearum inoculation done at 5 days post-anthesis (A through J); CK, uninoculated check (a through j). 


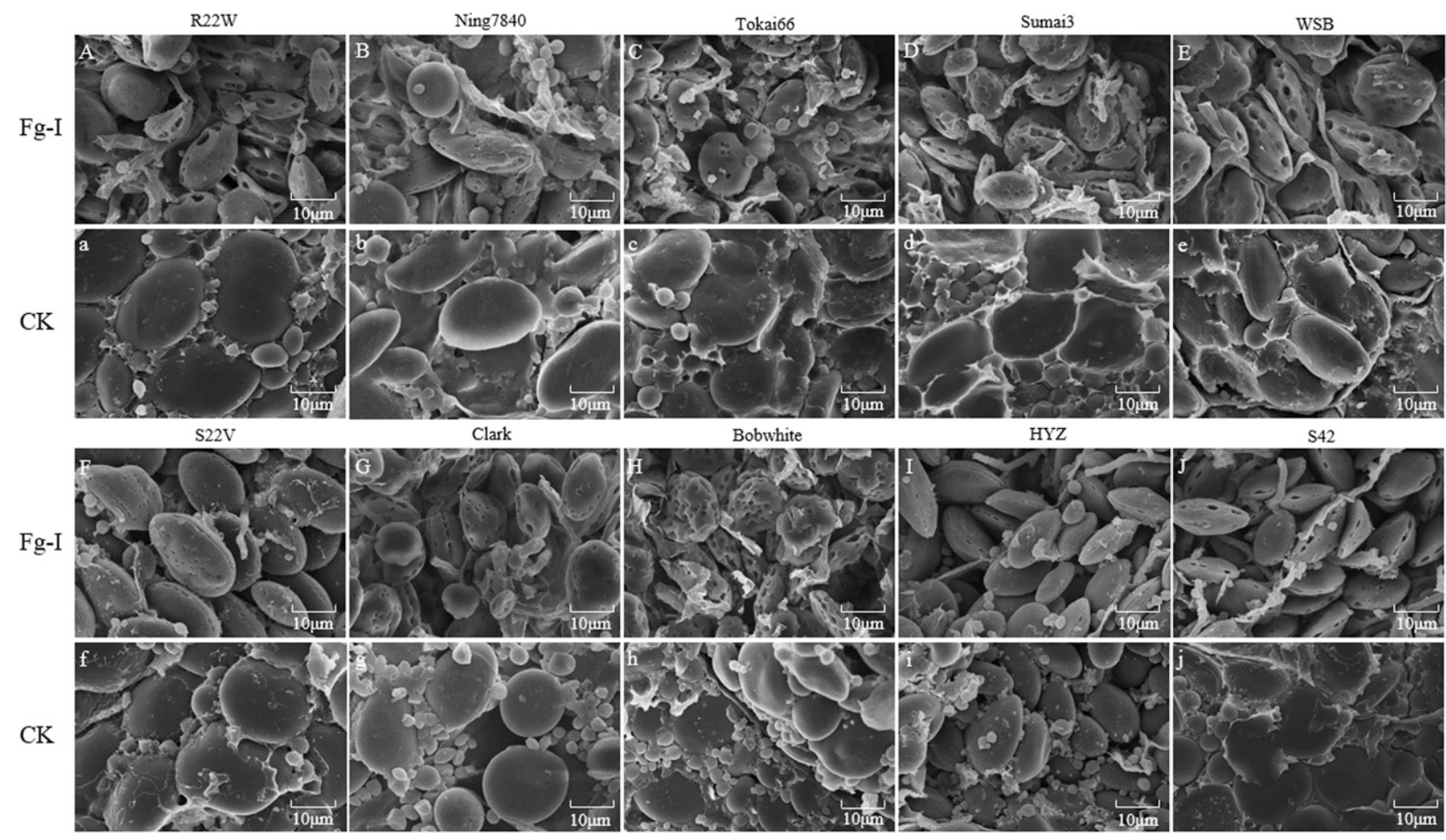

Fig. 3. Scanning electron microscopy of endosperm structures of inoculated genotypes sampled at 25 days postanthesis. A and a, R22W. B and b, Ning7840. C and c, Tokai66. D and d, Sumai3. E and e, Wangshuibai (WSB). F and f, S22V. G and g, Clark. H and h, Bobwhite. I and i, Haiyanzhong (HYZ). J and j, S42. Fg-l, Fusarium graminearum inoculation done at 10 days post-anthesis (A through J); CK, uninoculated check (a through j).
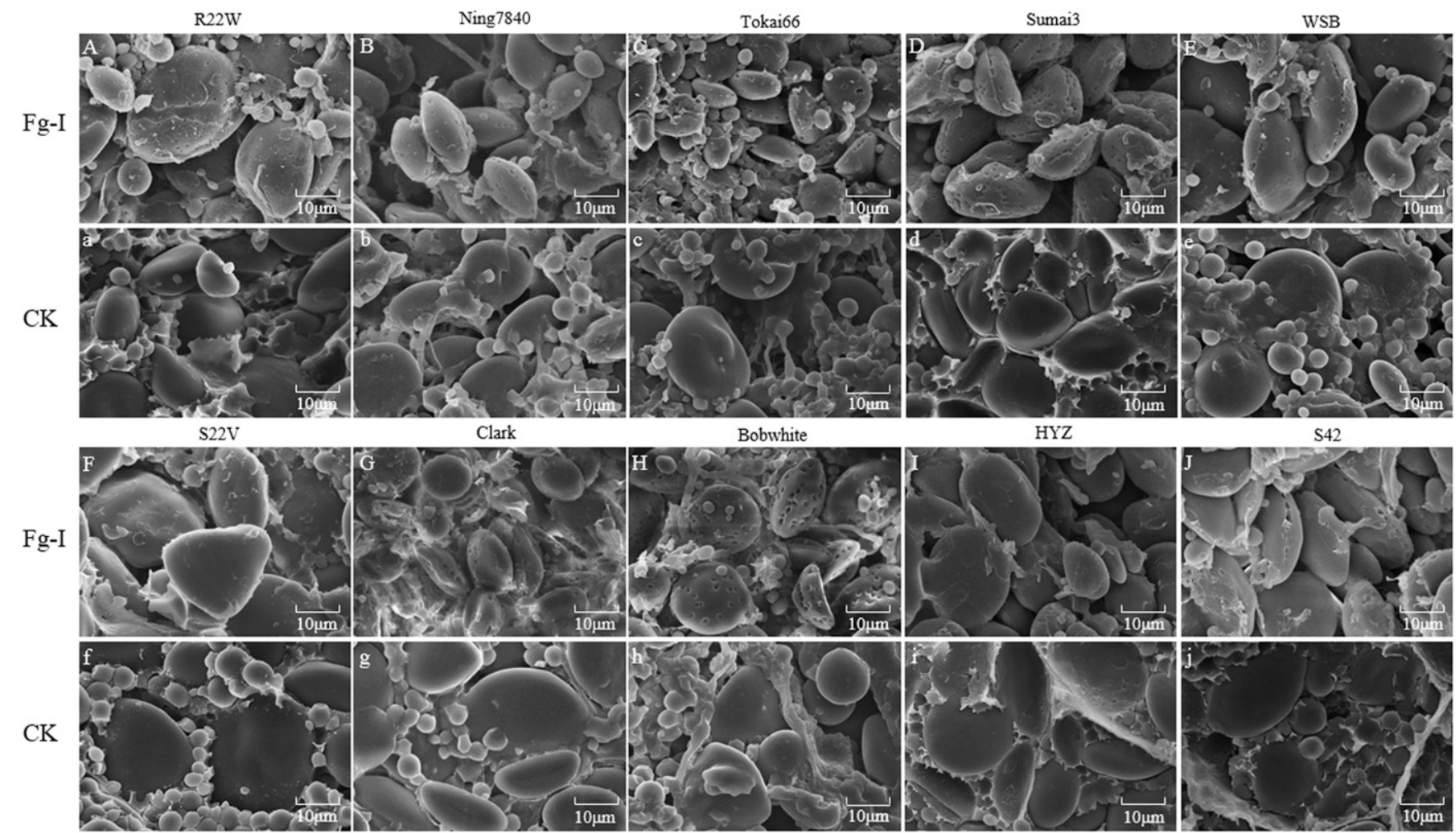

Fig. 4. Scanning electron microscopy of endosperm structures of inoculated genotypes sampled at 30 days postanthesis. A and a, R22W. B and b, Ning7840. C and c, Tokai66. D and d, Sumai3. E and e, Wangshuibai (WSB). F and f, S22V. G and g, Clark. $\mathrm{H}$ and h, Bobwhite. I and i, Haiyanzhong (HYZ). J and j, S42. Fg-l, Fusarium graminearum inoculation done at 15 days post-anthesis (A through J); CK, uninoculated check (a through j). 

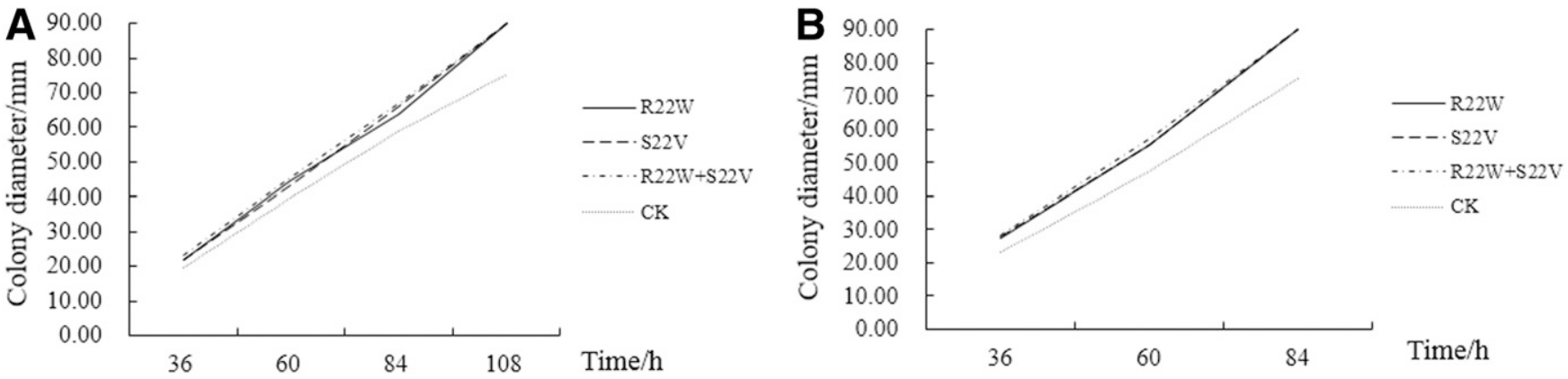

Fig. 5. Growth rate of Fusarium graminearum strains A, Fg0865 and B, FgW on potato dextrose agar medium with different treatments over the time course. CK, uninoculated check.

themselves. After a successful infection, enzymes are secreted from hyphae to destroy starch granules as well as grain protein (Ortega et al. 2019; Wang et al. 2005). With the aid of SEM, Packa et al. (2008) reported that small starch granules disappeared and large starch granules were severely damaged in Fusarium-infected kernels, which agrees well with our observations. However, no comparison was made on grain resistance between different genotypes in their study. In our experiments, regardless of inoculation timepoints, $\mathrm{S} 22 \mathrm{~V}$ without $F h b 1$ always showed less degradation in starch granules than R22W with $F h b 1$, implying that Fhb1 might have negative effects, or at least neutral effects, on grain resistance.

We were also wondering whether grain resistance against Fusarium infection varied among genotypes with different type II QTLs, ranging from resistant genotypes with or without $F h b l$ to susceptible genotypes without any known type II QTLs. Unexpectedly, we found that the Fhbl carrier Tokai66 with superior type II resistance turned out to be susceptible in terms of type IV resistance, whereas the nonFhb1 carrier HYZ exhibited good type IV resistance. These results clearly demonstrate that grain resistance does vary among genotypes, and it is independent of Fhbl and other QTL components mediating type II resistance.

In the in vitro experiment, no inhibition zone was observed in any of the three treatments; on the contrary, fungal colonies in plates with grains grew faster than those of the check, and grain samples at later developmental stages resulted in faster colony growth rates, implying that the accumulated carbon and nitrogen sources may stimulate fungal growth. The absence of a significant difference among the three treatments demonstrated that $F h b 1$ has no effect on in vitro grain resistance, in agreement with our in vivo experiments.

\section{Conclusion}

Here we proposed a new definition for grain resistance to Fusarium infection and developed an evaluation system for this type of resistance, including in vivo and in vitro experiments, in which different resistance levels were observed from the surface to the inner structure of grains. Our results demonstrated that grain resistance varies among wheat genotypes and is independent of type II resistance. We also demonstrated that Fhbl may have negative, or at least neutral, effects on grain resistance, further enriching knowledge on the function of this well-known QTL. We recommend merging type $\mathrm{V}$ into type IV resistance and using the reduction in TKW under the evaluation system proposed here as an indicator for type IV resistance, which simplified the FHB resistance types. However, whether our conclusions also apply to other FHB causal agents, such as $F$. culmorum, $F$. avenaceum, $F$. langsethiae, and $F$. sporotrichioides, remains to be clarified because only $F$. graminearum isolates of $15 \mathrm{ADON}$ chemotypes were used in this study.

\section{Literature Cited}

Abate, Z. A., Liu, S., and McKendry, A. L. 2008. Quantitative trait loci associated with deoxynivalenol content and kernel quality in the soft red winter wheat 'Ernie'. Crop Sci. 48:1408-1418.

Ágnes, S. H., Szabolcs, L. K., Monika, V., Laszlo, P., Janos, P., Csaba, L., and Akos, M. 2014. Differential influence of QTL linked to Fusarium head blight, Fusarium-damaged kernel, deoxynivalenol contents and associated morphological traits in a Frontana-derived wheat population [published correction appears in Euphytica 2014;200:27]. Euphytica 200:9-26.

Audenaert, K., De Boevre, M., Vanheule, A., Callewaert, J., Bekaert, B., Hofte, M., De Saeger, S., and Haesaert, G. 2013. Mycotoxin glucosylation in commercial wheat varieties: Impact on resistance to Fusarium graminearum under laboratory and field conditions. Food Control 34:756-762.

Bai, G. H., Su, Z. Q., and Cai, J. 2018. Wheat resistance to Fusarium head blight. Can. J. Plant Pathol. 40:336-346.

Balut, A. L., Clark, A. J., Brown-Guedira, G., Souza, E., and Van Sanford, D. A. 2013. Validation of Fhb1 and QFhs.nau-2DL in several soft red winter wheat populations. Crop Sci. 53:934-945.

Bonin, C., and Kolb, F. 2009. Resistance to Fusarium head blight and kernel damage in a winter wheat recombinant inbred line population. Crop Sci. 49:1304-1312.

Buerstmayr, H., Ban, T., and Anderson, J. A. 2009. QTL mapping and markerassisted selection for Fusarium head blight resistance in wheat: A review. Plant Breed. 128:1-26.

Clark, A. J., Sarti-Dvorjak, D., Brown-Guedira, G., Dong, Y., Baik, B. K., and Van Sanford, D. A. 2016. Identifying rare FHB-resistant segregants in intransigent backcross and F2 winter wheat populations. Front. Microbiol. 7:277.

Foroud, N. A., Baines, D., Gagkaeva, T. Y., Thakor, N., Badea, A., Steiner, B., Burstmayr, M., and Burstmayr, H. 2019. Trichothecenes in cereal grains - an update. Toxins (Basel) 11:634.

Goral, T., Wisniewska, H., Ochodzki, P., Nielsen, L. K., Walentyn-Goral, D., and Stepien, L. 2019. Relationship between Fusarium head blight, kernel damage, concentration of Fusarium biomass, and Fusarium toxins in grain of winter wheat inoculated with Fusarium culmorum. Toxins (Basel) 11:2.

Hofstad, A. N., Nussbaumer, T., Akhunov, E., Shin, S., Kugler, K. G., Kistler, H. C., Mayer, K. F. X., and Muehlbauer, G. J. 2016. Examining the transcriptional response in wheat Fhb1 near-isogenic lines to Fusarium graminearum infection and deoxynivalenol treatment. Plant Genome 9:1-15.

Islam, M. S., Brown-Guedira, G., Van Sanford, D., Ohm, H., Dong, Y. H., and McKendry, A. L. 2016. Novel QTL associated with the Fusarium head blight resistance in Truman soft red winter wheat. Euphytica 207:571-592.

Jansen, C., von Wettstein, D., Schafer, W., Kogel, K. H., Felk, A., and Maier, F. J. 2005. Infection patterns in barley and wheat spikes inoculated with wild-type and trichodiene synthase gene disrupted Fusarium graminearum. Proc. Natl. Acad. Sci. USA 102:16892-16897.

Lemmens, M., Koutnik, A., Steiner, B., Buerstayr, H., Berthiller, F., Schuhmacher, R., Maier, F., and Schafer, W. 2008. Investigations on the ability of FHB1 to protect wheat against nivalenol and deoxynivalenol. Cereal Res. Commun. 36(suppl 6):429-435.

Li, G. Q., Zhou, J. Y., Jia, H. Y., Gao, Z. X., Fan, M., Luo, Y. J., Zhao, P. T., Xue, S. L., Li, N., Yuan, Y., Ma, S. W., Kong, Z. X., Jia, L., An, X., Jiang, G., Liu, W. X., Cao, W. J., Zhang, R. R., Fan, J. C., Xu, X. W., Liu, Y. F., Kong, Q. Q., Zheng, S. H., Wang, Y., Qin, B., Cao, S. Y., Ding, Y. X., Shi, J. X., Yan, H. S., Wang, X., Ran, C. F., and Ma, Z. Q. 2019. Mutation of a histidine-rich calciumbinding-protein gene in wheat confers resistance to Fusarium head blight. Nat. Genet. 51:1106-1112.

Liu, S. Y., Hall, M. D., Griffey, C. A., and McKendry, A. L. 2009. Meta-analysis of QTL associated with Fusarium head blight resistance in wheat. Crop Sci. 49: 1955-1968.

Mesterházy, A. 1995. Types and components of resistance to Fusarium head blight of wheat. Plant Breed. 114:377-386.

Mesterházy, A., Bartok, T., Mirocha, C. G., and Komoroczy, R. 1999. Nature of wheat resistance to Fusarium head blight and the role of deoxynivalenol for breeding. Plant Breed. 118:97-110.

Ortega, L. M., Moure, M. C., Gonzalez, E. M., and Alconada, T. M. 2019. Wheat storage proteins: Changes on the glutenins after wheat infection with different isolates of Fusarium graminearum. Int. Microbiol. 22:289-296.

Packa, D., Jackowiak, H., Góral, T., Wiwart, M., and Perkowski, J. 2008. Scanning electron microscopy of Fusarium-infected kernels of winter triticale (x Triticosecale Wittmack). Seed Sci. Biotechnol. 2:27-31.

Parker, M. L. 1985. The relationship between A-type and B-type starch granules in the developing endosperm of wheat. J. Cereal Sci. 3:271-278. 
Rawat, N., Pumphrey, M. O., Liu, S., Zhang, X., Tiwari, V. K., Ando, K., Trick, H. N., Bockus, W. W., Akhunov, E., Anderson, J. A., and Gill, B. S. 2016. Wheat Fhb1 encodes a chimeric lectin with agglutinin domains and a poreforming toxin-like domain conferring resistance to Fusarium head blight. Nat. Genet. 48:1576-1580.

Schroeder, H. W., and Christensen, J. J. 1963. Factors affecting resistance of wheat to scab caused by Gibberella zeae. Phytopathology 53:831-838.

Schweiger, W., Steiner, B., Vautrin, S., Nussbaumer, T., Siegwart, G., Zamini, M., Jungreithmeier, F., Gratl, V., Lemmens, M., Mayer, K. F. X., Berges, H., Adam, G., and Buerstmayr, H. 2016. Suppressed recombination and unique candidate genes in the divergent haplotype encoding Fhb1, a major Fusarium head blight resistance locus in wheat. Theor. Appl. Genet. 129:1607-1623.

Su, Z., Bernardo, A., Tian, B., Chen, H., Wang, S., Ma, H., Cai, S., Liu, D., Zhang, D., Li, T., Trick, H., St. Amand, P., Yu, J., Zhang, Z., and Bai, G. 2019. A deletion mutation in TaHRC confers Fhbl resistance to Fusarium head blight in wheat. Nat. Genet. 51:1099-1105.
Szabó-Hevér, A., Lehoczki-Krsjak, S., Toth, B., Purnhauser, L., Buerstmayr, H. Steiner, B., and Mesterházy, A. 2012. Identification and validation of Fusarium head blight and Fusarium-damaged kernel QTL in a Frontana/Remus DH mapping population. Can. J. Plant Pathol. 34:224-238.

Tamburic-Ilincic, L. 2012. Effect of 3B, 5A and 3A QTL for Fusarium head blight resistance on agronomic and quality performance of Canadian winter wheat Plant Breed. 131:722-727.

Walter, S., Kahla, A., Arunachalam, C., Perochon, A., Khan, M. R., Scofield, S. R., and Doohan, F. M. 2015. A wheat ABC transporter contributes to both grain formation and mycotoxin tolerance. J. Exp. Bot. 66:2583-2593.

Wang, J. H., Wieser, H., Pawelzik, E., Weinert, J., Keutgen, A. J., and Wolf, G. A. 2005 Impact of the fungal protease produced by Fusarium culmorum on the protein quality and breadmaking properties of winter wheat. Eur. Food Res. Technol. 220:552-559.

Yang, Z., Gilbert, J., Fedak, G., and Somers, D. J. 2005. Genetic characterization of QTL associated with resistance to Fusarium head blight in a doubled-haploid spring wheat population. Genome 48:187-196. 